\title{
Vectorcardiographic features of ventricular extrasystoles correlated with conventional scalar electrocardiographic interpretation
}

\author{
S. TALBOT, D. KILPATRICK, AND B. WEEKS
}

From the Division of Cardiovascutar Disease, Royal Postgraduate Medical School, Hammersmith Hospital, Ducane Road, London

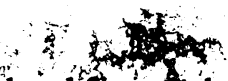

SUMMARY Horizontal and frontat plane QRS loops of patients in sinus rhythm with uniform ventricular extrasystoles were constructed from digitised Frank orthogonal electrocardiograms.

In 4 patients ventricular extrasystoles were indistinguishable from right bundle-branch complexes, and in another they were indistinguishable from left bundle-branch complexes. In 25 patients ventricular extrasystoles showed an initial delay ( $\geq 20 \mathrm{~ms}$ ) of the $Q R S$, followed by an $R$ loop, which in 13 patients resembled left bundle-branch block complexes, and in 12 patients resembled right bundle-branch block complexes, with an anterior clockwise loop in the horizontal plane. However, the frontal plane loop often did not resemble that of either right or left bundle-branch block. In 51 patients ventricular extrasystoles had an initial delay which was prolonged into the efferent limb of the QRS loop with acceleration of the afferent limb, and/or the QRS loop was directed anteriorly, inferiorly, and to the left. Conventional recordings of these extrasystoles usually showed an initial slow upstroke (or downstroke) of the QRS resembling a delta wave.

Apparently uniform ventricular extrasystoles on scalar recordings were shown to be multiform vectorcardiographically though in all such cases the direction of the initial $40 \mathrm{~ms}$ forces was constant.

It is suggested that the slow initial inscription of ventricular extrasystoles is the result of excitation of ventricular muscle directly and not through specialised His-Purkinje fibres, and that the direction of such initial forces may indicate the ventricular origin of ventricular extrasystoles.

The form of ventricular extrasystoles has been studied for many years (Scherf and Schott, 1973). Such studies were limited because simultaneous recordings in three planes were then impossible (Rosenbaum, 1969). Description of the shape of ventricular extrasystoles in the 12 lead electrocardiogram requires simultaneous recording of all the leads, which is difficult to achieve. Earlier studies of ventricular extrasystoles by the Frank orthogonal system overcame these difficulties (Talbot and Dreifus, 1975), but precise description of the direction of the initial and terminal forces is best shown by vectorcardiograms derived from these leads.

Vectorcardiograms of ventricular extrasystoles have been described in dogs (Boineau et al., 1960) and in man (Sano et al., 1967). However, extrasystoles are usually unpredictable and arise close to

Received for publication 10 October 1977. preceding conducted beats, so that either recordings of the initial forces have been of poor quality or only ventricular bigeminy has been studied (Zoneraich and Zoneraich, 1972). In the latter condition extrasystoles arise equally close to preceding conducted beats and can be anticipated satisfactorily. Vectorcardiographic recording of the QRS waveform of tachyarrhythmias is even more difficult. Benchimol (1973) tried to overcome these difficulties by recording timed vectorcardiograms, but because of the moving time base these loops were distorted.

We have been able to isolate vectorcardiograms of ventricular extrasystoles from recordings of Frank orthogonal electrocardiograms on electromagnetic tape using a PDP 12 digital computer. Similar methods have been used by Klein et al. (1976) to show the vectorial characteristics of extrasystoles induced during cardiac catheterisation. However this research was not directed at the initial 
and terminal forces of the QRS, which probably indicate the origin of arrhythmias and may help differentiate aberrant conduction from ventricular arrhythmias. This was the aim of the present study. Ultimately these results will be correlated with epicardial mapping of ventricular and supraventricular arrhythmias.

\section{Subjects and methods}

Frank orthogonal electrocardiograms of 59 men and 25 women in sinus rhythm with 3 or more uniform ventricular extrasystoles were recorded on paper and on electromagnetic tape. Ventricular extrasystoles of 21 men and 3 women were recorded only during or immediately after exercise. Later $X$, $\mathrm{Y}$, and $\mathrm{Z}$ leads were displayed on the oscilloscope of a PDP 12 computer and stored. Ventricular extrasystoles which were located after the end of the $T$ wave of the preceding conducted beat, but before the next $P$ wave, were identified and the onset and offset of each QRS were marked with a cursor. The QRS complexes of each extrasystole and the preceding sinus beat were digitised separately. Later XY (frontal) and XZ (horizontal) plots were constructed from the digital recordings using a Complot DPI digital XY plotter so that the frontal and horizontal plane vectorcardiograms were displayed at maximum magnification. The clinical features of each patient were recorded.

The vectorcardiographic loops were analysed by the method of Witham (1975). The first change of direction in both planes is called the $Q$ point; the second turning point (the apex of the loop) is the $R$ point; if there is another point of inflection then this is called the $S$ point and the forces after this the $S$ loop. In addition QRS loops can be divided into initial, mid-zone, and terminal forces on the basis of the tangential velocity of inscription of the QRS in both planes. In normal people the forces up to the $Q$ point usually correspond to a phase of relatively slow inscription, the loop up to the $R$ point to a phase of more rapid inscription, and the $S$ loop to a phase of terminal slowing.

Initial slow forces were usually obvious because they were followed by distinct acceleration in two planes of the efferent limb of the vectorcardiogram. However there were exceptions and therefore the time at which the speed of inscription of the QRS of the extrasystole reached the same speed as that of the normal QRS after the q loop or initial delay was taken as the duration of the initial forces. When there was a difference in the duration of the initial forces in the two planes the mean duration was determined.

Vectorcardiographic loops of the ventricular

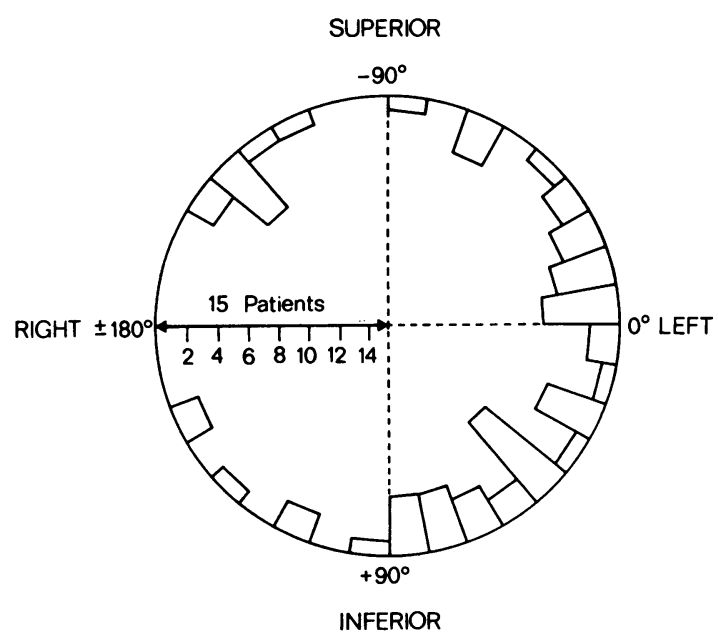

Fig. 1 Direction of the $R$ point in 63 ventricular extrasystoles. (Frontal plane).

extrasystoles were compared with the normal QRS of each patient and with the vectorcardiographic appearance of right bundle-branch block and left bundle-branch block (Witham, 1975). When possible 12 lead electrocardiograms of apparently uniform ventricular extrasystoles were recorded at the same time.

\section{Results}

Of the 84 patients studied, 3 had left anterior hemiblock, 2 had non-specific ventricular conduction defects, 2 had right bundle-branch block, and

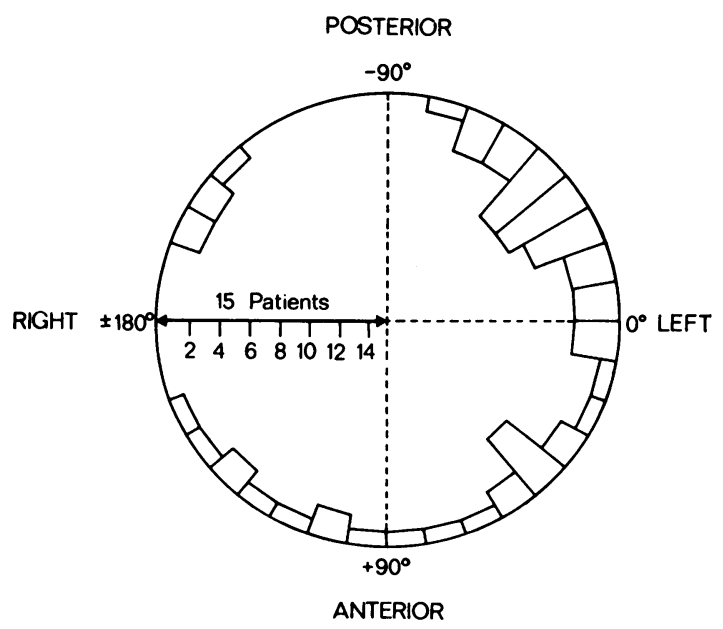

Fig. 2 Direction of the $R$ point in 61 ventricular extrasystoles (horizontal plane). 


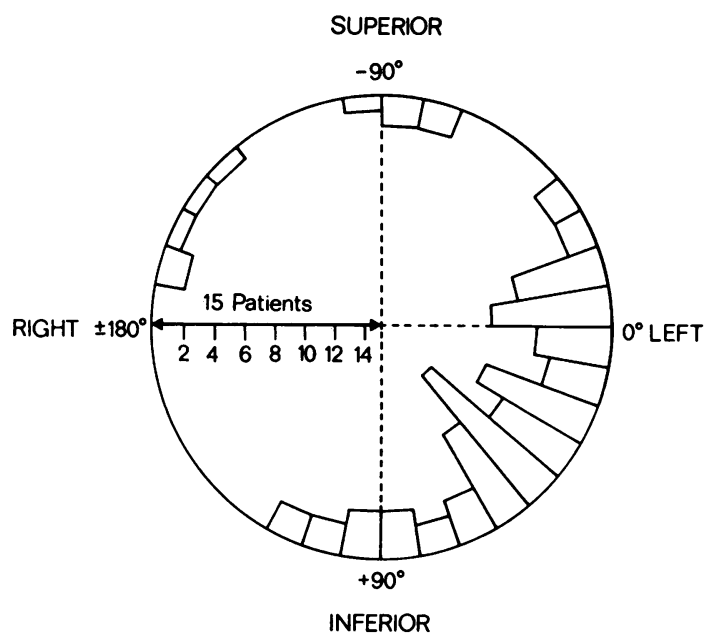

Fig. 3 Direction of the initial forces (frontal plane).

1 had left bundle-branch block by conventional criteria (Talbot et al., 1976).

The direction of the $\mathbf{R}$ point of the ventricular extrasystoles is shown in Fig. 1 and 2. Because the $\mathbf{R}$ point was not distinct and the main loop appeared biphasic, 21 loops are not included in Fig. 1 and 23 loops are not included in Fig. 2.

The direction of the initial forces is shown in Fig. 3 and 4. They were usually directed to the left anteriorly and inferiorly, whereas the $R$ point was more variable, either being directed posteriorly or anteriorly though usually to the left and inferiorly. There were 5 extrasystoles which did appear to be a separate group, since the initial forces and the $\mathbf{R}$ point were directed to the right

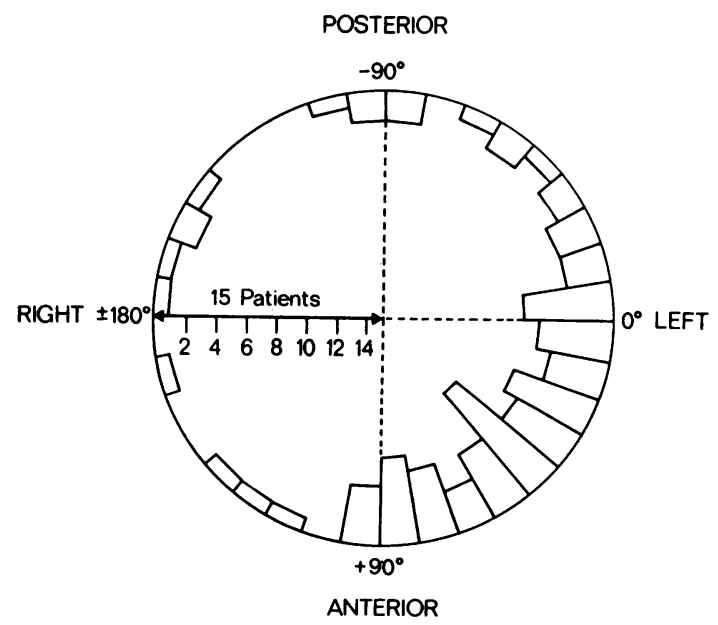

Fig. 4 Direction of the initial forces (horizontal plane). and superiorly; usually the $\mathbf{R}$ point of these extrasystoles was somewhat posterior in the horizontal plane.

In order to see if ventricular extrasystoles resembled either left or right bundle-branch block each extrasystole was compared with vectorcardiograms of these blocks. The vectorcardiographic features of left bundle-branch block are minimal or there are no anterior initial forces; initial forces are usually directed to the left and last less than $20 \mathrm{~ms}$, and are followed by a more rapid inscription posteriorly and slightly to the left nearly to the $R$ point, but this phase is never as rapid as the efferent limb of the normal $Q R S$. There is a midzone delay at the $R$ point, with variable delay of the afferent limb which is also directed posteriorly and usually slightly to the left. In the frontal plane the loop is small and directed to the left. It may be either superior or inferior. There were 4 vectorcardiograms of ventricular extrasystoles which could be considered to show such features; in addition ventricular extrasystoles of another 13 patients resembled left bundle-branch complexes except for prolonged initial forces. There were usually more anterior forces with slow inscription before the rapid phase directed posteriorly, and these slow initial forces lasted more than $20 \mathrm{~ms}$. In 2 of these extrasystoles the second part of the QRS was ill defined and the loops had a generalised slow inscription. In 1 patient with an anteroseptal infarction, initial forces of the extrasystole were directed clearly to the right and anteriorly with a clockwise loop, as has been shown before with left bundle-branch block and anteroseptal infarction. Of the 4 ventricular extrasystoles 3 had left axis deviation and of the 13, 5 had left axis deviation.

The vectorcardiographic features of right bundlebranch block are a brief initial q loop usually directed to the right and anteriorly; the initial forces last less than $20 \mathrm{~ms}$. The efferent limb is directed to the left and then either there is some posterior orientation with the afferent limb crossing the efferent limb as it is directed to the right and anteriorly (type $A$ ) or the $R$ loop is directed anteriorly, with a clockwise direction ending in terminal forces on the right (type B) (Kulbertus et al., 1976). In both types there is terminal delay anteriorly or slightly posterior and to the right. There were 4 vectorcardiograms of extrasystoles that had such features and all showed either right or left axis deviation. Ventricular extrasystoles of another 12 patients showed an initial delay of at least $20 \mathrm{~ms}$ compared with the basic QRS followed by anterior QRS loops which could resemble right bundle-branch block (Fig. 5). It should be noted however that in only 2 patients were the loops 


\section{HORIZONTAL}

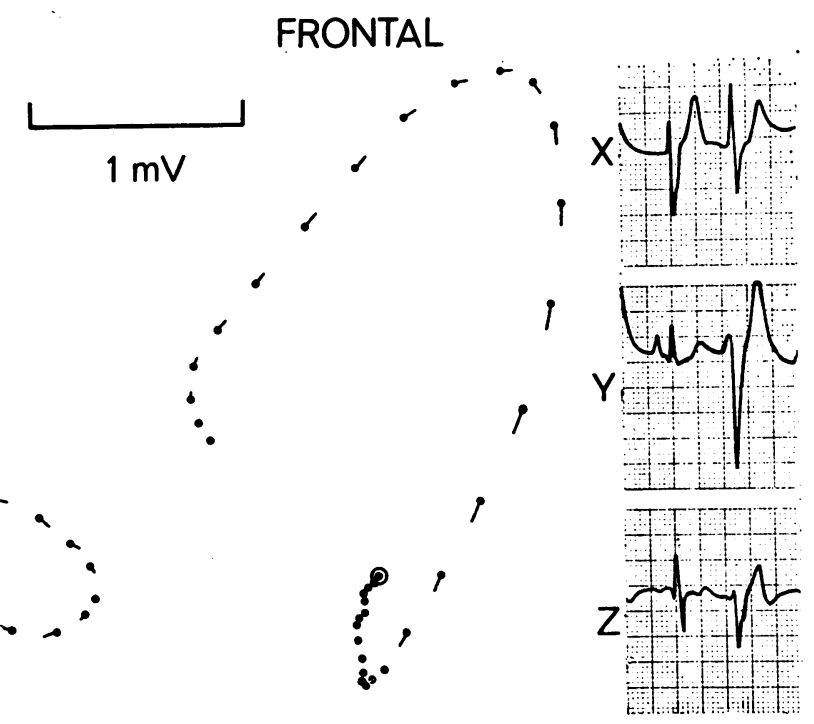

Fig. 5 Vectorcardiogram and electrocardiogram. There are slow initial forces but the rest of the QRS loop is similar to a right bundle-branch complex. The frontal plane axis is directed superiorly.

counterclockwise (like type $A$ ). In addition, the terminal delay was not as pronounced as in typical right bundle-branch complexes, and initial forces were not always directed anteriorly and from left to right. In 3 patients there was generalised delay of QRS inscription. Right bundle-branch block often has a biphasic QRS loop and this group of extrasystoles was similar; when the main QRS loop could be identified it showed either right or left axis deviation in 8 patients.

However, the majority of ventricular extrasystoles were dissimilar to bundle-branch complexes. Forty patients showed a slow initial inscription lasting at least $40 \mathrm{~ms}$, that is it took at least $40 \mathrm{~ms}$ for the tangential velocity of inscription of the QRS loop of the extrasystole to reach the speed of inscription

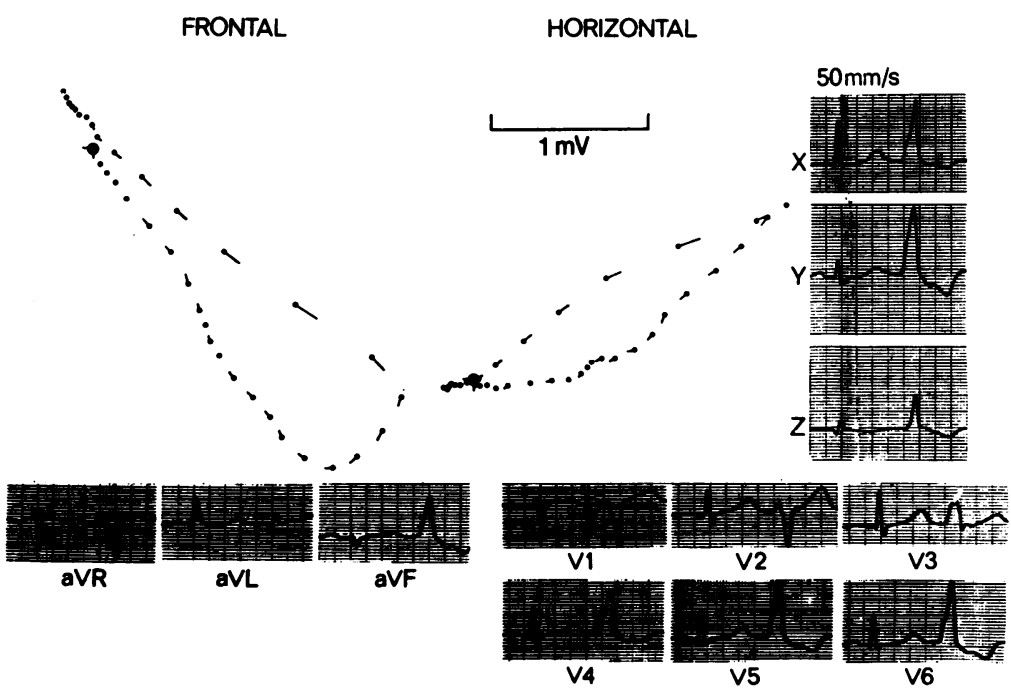

Fig. 6 Ventricular extrasystole with initial delay prolonged into the efferent limb of the extrasystole. 
(A)

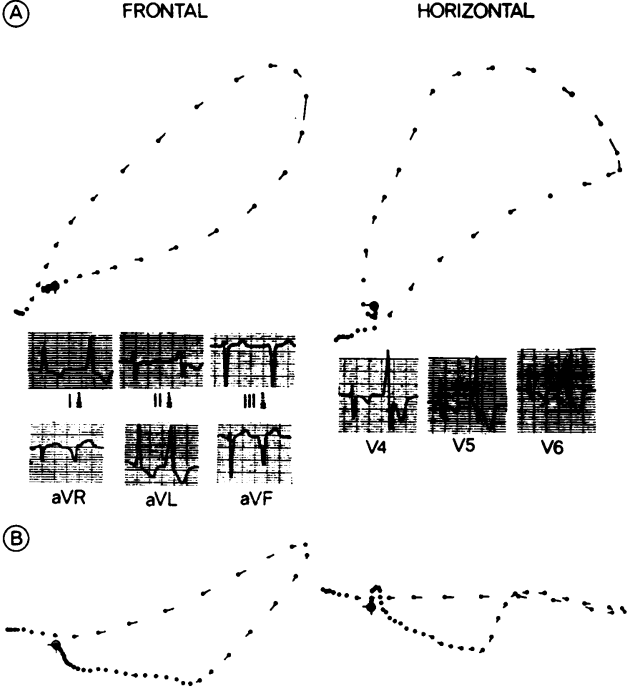

Fig. 7 A. $Q R S$ of a normal sinus beat with left anterior hemiblock and old anterolateral infarction. B. $Q R S$ of ventricular extrasystole with prolonged initial forces demarcated from the efferent limb of the $Q R S$.

of the normal QRS loop at the end of the $Q$ loop; if there was an obvious $Q$ loop to the extrasystole, this was at least $40 \mathrm{~ms}$ in duration. Thus, in 25 of these patients the initial delay in the ventricular extrasystole was not clearly demarcated from the efferent limb of the $R$ loop (Fig. 6) but in the remainder such a demarcation was obvious (Fig. 7), and in 8 patients there was a clear change of direction in both planes at this point. In general the ventricular extrasystoles showed a monophasic QRS in the frontal plane and sometimes in the horizontal plane. In these 40 patients the initial forces were directed predominantly inferiorly, anteriorly, and to the left. However in 20 patients the $R$ point was directed posteriorly and to the left.

In a further 11 patients the initial forces were shorter ( $\geqslant 20 \mathrm{~ms} \leqslant 40 \mathrm{~ms}$ ) but the principal QRS loop and the $R$ point were directed anteriorly, inferiorly, and to the left. This distinguished these ventricular extrasystoles from bundle-branch complexes; in addition the QRS loop when entirely anterior had a counterclockwise inscription.

These 51 patients with distinctive QRS waveform therefore, account for the predominant directions of initial and maximal QRS forces shown in Fig. 1 to 4 . In these extrasystoles terminal forces had variable duration and speed of inscription merging with the ST segment. An S loop was uncommon.

Although all extrasystoles were apparently uniform on the 3 simultaneous leads of the scalar electrocardiogram, the main QRS loop, and sometimes the $R$ point were not entirely uniform on the vectorcardiogram. The initial $20 \mathrm{~ms} Q R S$ forces in all these vectorcardiograms were the same (Fig. 8) but the contour of the loop changed at variable

FRONTAL

HORIZONTAL
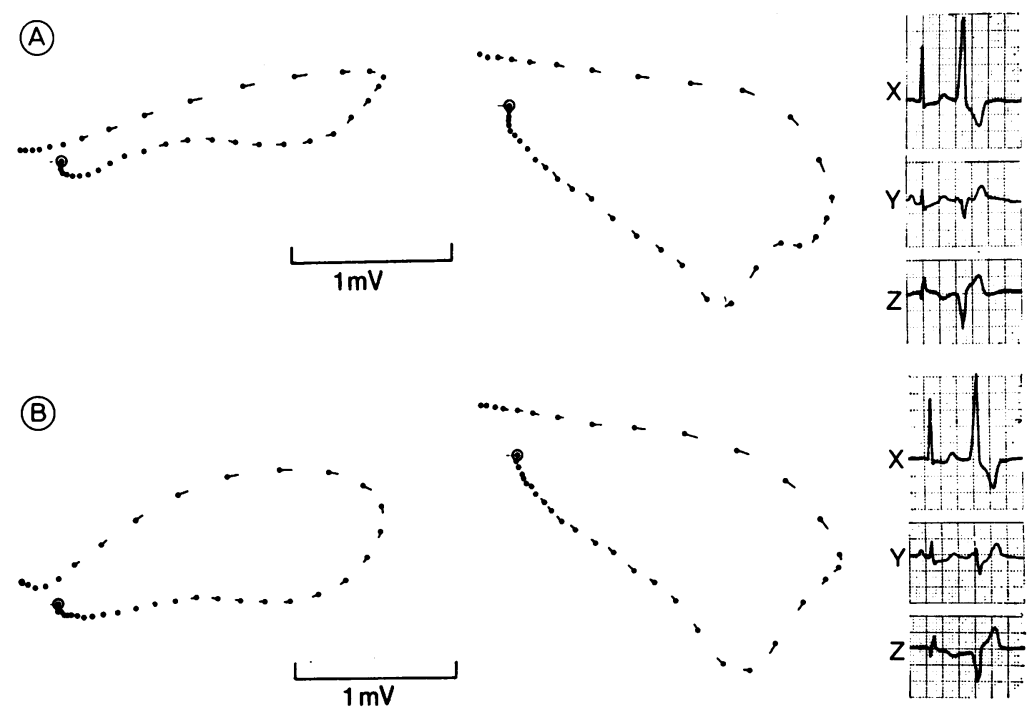

Fig. 8 Apparently uniform ventricular extrasystoles on scalar electrocardiograms $A$ and $B$. However, the corresponding vectorcardiograms show some multiformity though the initial $Q R S$ forces are constant. 
FRONTAL

HORIZONTAL

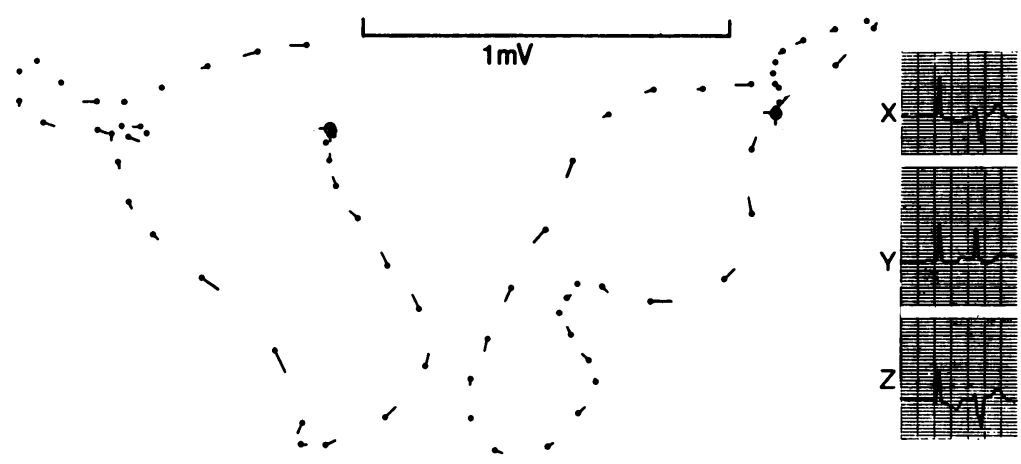

Fig. 9 Bizarre shape of ventricular extrasystole with initial $Q R S$ delay and right bundle-branch type complex in a patient with old anterior infarction.

points after this. In 5 patients this may have been the result of varying prematurity of ventricular extrasystoles, though in a further 15 patients (out of 74) such varying prematurity was not associated with any variability of the vectorcardiographic QRS loop.

Display of the scalar representation of the maximum spatial $Q R S$ voltage showed that the $Q R S$ voltage of the ventricular extrasystoles was greater than the basic QRS in all but 6 patients. Because the majority of extrasystoles were orientated inferiorly, the discrepancy in QRS voltage was usually most evident in the frontal plane. Ventricular extrasystoles in which the initial forces were orientated in the same direction as the maximum spatial QRS often showed a characteristic step on the upstroke of the QRS resembling a delta wave, before the rapid rise of voltage to the peak of the $R$ wave.

There were no differences between the extrasystoles at rest and those on or after exercise. Ventricular tachycardia occurred in 3 patients, 2 on exercise. These tachycardias had a similar form to the uniform ventricular extrasystoles.

Ventricular extrasystoles were found in 6 patients with old anterior infarction. Because of the usual direction of initial forces of extrasystoles anteriorly, anterior infarction on the electrocardiogram was sometimes concealed, but in 3 patients initial forces were directed posteriorly, with later forces directed anteriorly (Fig. 9). Ventricular extrasystoles were found in 9 patients with inferior infarction. Inferior infarction pattern was concealed in 4 patients because initial forces of extrasystoles are usually directed inferiorly. However, in 5 patients the initial forces of the extrasystole were directed horizontally or superiorly. Of the 33 patients with extrasystoles showing features of either right or left bundle-branch complexes, 26 had ischaemic heart disease.

\section{Discussion}

This study has shown that the majority of uniform ventricular extrasystoles do not resemble bundlebranch complexes. The large monophasic QRS complexes orientated inferiorly in the frontal plane have their counterpart in the monophasic QRS complexes in leads II, III, and aVF. The frequent anterior orientation in the horizontal plane will produce dominant monophasic RR or Rsr complexes in V1 to V3, which Sandler and Marriott (1965) described as typical of ventricular ectopy. However, if there is a clockwise loop there may be enough orientation of the efferent limb of the QRS to the left for a $\mathrm{qR}$ complex to be found in V1. If there is a counterclockwise loop orientated anteriorly in the horizontal plane an rsR complex may be seen in V1. When this lead has these appearances it may be confused with aberration. In addition because superior and inferior orientation of the QRS will affect the appearance of V1 and V6, these well-known 12 lead features of aberration and ectopy may be inaccurate.

Extrasystoles are sometimes difficult to classify as either left or right bundle-branch block type from the scalar electrocardiogram though this is easier if the directions of the terminal forces on the orthogonal electrocardiogram are examined (Talbot and Dreifus, 1975). The total QRS direction is often distinctive and initial delay is frequent; this initial delay may be seen on the scalar electrocardiogram because it is usually directed anteriorly, inferiorly, and to the left; thus, inferior limb and left anterior praecordial leads will usually show a 
step at the origin of the QRS. This is similar to the delta wave of Wolff-Parkinson-White syndrome. It may indicate the direction of spread of ventricular depolarisation from an origin in the ventricular myocardium. Therefore, it may be possible to determine the site of origin of ventricular extrasystoles just as it is possible to determine the insertion of an anomalous bypass from the spatial direction of the delta wave.

The initial delay has been described before as a universal feature of ventricular extrasystoles (Zoneraich and Zoneraich, 1972), or the delay has not been commented on (Sano et al., 1967). It does not appear to be universal, but may be a useful differentiating point from aberrant conduction. Later QRS forces of ventricular extrasystoles probably also indicate the ventricle that has been prematurely stimulated and the axis changes of such extrasystoles may indicate whether inflow, outflow, or apical ventricular regions have been initially excited or which fascicle has been excited first (Rosenbaum, 1969; Klein et al., 1976). Preferential excitation of one fascicle may well explain the distinct axis changes of the ventricular extrasystoles with bundle-branch features. The initial delay if present could represent the spread of excitation in non-specialised fibres before a neighbouring fascicle is excited. Then if the left bundle-branch system is excited a right bundle-branch complex is produced and vice versa; preferential excitation of the left anterior fascicle would produce a vertical or right axis (Rosenbaum, 1969; Talbot and Dreifus, 1975) However, because the septum may form an important source of ventricular arrhythmias (particularly in ischaemic heart disease) and the main fascicles are in close proximity to ventricular foci on either side of the septum, the bundle-branch configuration may not adequately localise the ventricular origin of any extrasystole.

The most characteristic appearance of ventricular extrasystoles-namely the initial delay-is followed by faster QRS inscription. The reason for this is not clear since it is not the result of ventricular fusion with a normally conducted supraventricular beat as occurs in Wolff-Parkinson-White syndrome. It may indicate later variable excitation of specialised conducting pathways so that some ventricular muscle is excited rapidly; certainly distinct bundlebranch complexes cannot be identified. Zoneraich and Zoneraich (1972) suggested that ventricular extrasystoles in patients with ischaemic heart disease were different from those in normal people, but they were only studying ventricular bigeminy. In this study the bizarre shapes of 'ischaemic' ventricular extrasystoles were seen occasionally (Fig. 9).

Vectorcardiography has been shown to identify clearly the direction of ventricular excitation in Wolff-Parkinson-White syndrome, and to aid interpretation of the delta wave on the scalar electrocardiogram. Delta waves are accentuated if either the initial direction or speed of depolarisation is different from later depolarisation, and the waves are minimised if these are similar when the delta wave only appears as a slower initial than later inscription of the upstroke (or downstroke) of the QRS or a generalised slow upstroke. Appearances simulating the delta waves of the Wolff-ParkinsonWhite syndrome but without pre-excitation may also be seen if the initial forces are directed nearly perpendicular to the direction of the recording electrode; likewise they may be absent in some leads in definite pre-excitation if initial forces are directed strictly tangentially (when the recording is isoelectric and the origin of the QRS may be missed). Similar considerations apply to ventricular extrasystoles; initial delay on the scalar electrocardiogram must be seen in multiple leads recorded in different directions and these leads must be recorded simultaneously. Superficial examination of bundlebranch configurations may show delta waves; for example, left bundle-branch complexes show initial delay in the frontal plane, and the whole QRS has slow inscription; however, in the horizontal plane there is no initial delay and therefore 'delta waves' will be seen only in the limb leads and V6 which is perpendicular to $-90^{\circ}$ (the usual posterior direction of the QRS).

Vectorcardiography helps the clinician to avoid these pitfalls and emphasises the similarities between most ventricular arrhythmias and preexcitation and their differences from aberrant conduction. It is known that three simultaneous orthogonal leads are necessary to differentiate uniform from multiform QRS complexes; however, if our preliminary results are confirmed multiformity of ventricular extrasystoles may have to be defined vectorially and varying initial forces taken as a criterion of multifocal nature. Ventricular extrasystoles with classical bundle-branch configuration may arise in the bundle-branches or the His bundle with distal aberration and may have a different significance from extrasystoles arising in ventricular myocardium or at the Purkinje myocardial junction.

\section{References}

Benchimol, A. (1973). Vectorcardiography, pp. 162-195. Williams and Wilkins, Baltimore.

Boineau, J. P., Spach, M. S., and Harris, J. S. (1960). Study of premature systoles of the canine heart by means of the spatial vectorcardiogram. American Heart fournal, 60, 924-935. 
Klein, M. D., Feldman, C. L., Clark, D. L., Flessas, A. P. Ryan, T. J., and Peura, R. A. (1976). Vectorial characteristics of ventricular extrasystoles stimulated during cardiac catheterisation. Fournal of Electrocardiology, 9, 103-108.

Kulbertus, H. E., de Leval-Rutten, F., and Casters, P. (1976). Vectorcardiographic study of aberrant conduction. British Heart fournal, 38, 549-557.

Rosenbaum, M. B. (1969). Classification of ventricular extrasystoles according to form. Fournal of Electrocardiology, 2, 289-298.

Sandler, J. A., and Marriott, H. J. L. (1965). The differential morphology of anomalous ventricular complexes of RBBB-type in lead $V_{1}$ : ventricular ectopy versus aberration. Circulation, 31, 551-556.

Sano, T., Hiroki, T., and Suzuki, F. (1967). The vectorcardiogram of ventricular premature beats. An analysis of vectorcardiograms from 100 cases taken by Frank's method. Fapanese Heart fournal, 8, 388-400.

Scherf, D., and Schcit, A. (1973). Extrasystoles and Allied Arrhythmias, 2nd ed., p. 542. Heinemann Medical Books, London.
Talbot, S., and Dreifus, L. S. (1975). Characteristics of ventricular extrasystoles and their prognostic importance. Chest, 67, 665-674.

Talbot, S., Dreifus, L. S., Watanabe, Y., Chiang, R., Morris, K., and Reich, M. (1976). Diagnostic criteria for computeraided electrocardiographic 15-lead system; evaluation using 12 leads and Frank orthogonal leads with vector display. British Heart Fournal, 38, 1247-1261.

Witham, A. C. (1975). A System of Vectorcardiographic Interpretation, p. 28. Year Book Medical Publishers, Chicago.

Zoneraich, O., and Zoneraich, S. (1972). Bigeminal rhythm vectorcardiographic patterns in coronary artery disease. Fournal of Electrocardiology, 5, 265-272.

Requests for reprints to Dr S. Talbot, Division of Cardiovascular Disease, Royal Postgraduate Medical School, Hammersmith Hospital, Ducane Road, London W12 0HS. 